\title{
Crustal and time-varying magnetic fields at the InSight landing site on Mars
}

\author{
Catherine L. Johnson ${ }^{1,2} \bowtie$, Anna Mittelholz' ${ }^{1}$, Benoit Langlais ${ }^{3}$, Christopher T. Russell ${ }^{4}$, \\ Véronique Ansan $\mathbb{1}^{3}$, Don Banfield ${ }^{1}{ }^{5}$, Peter J. Chi ${ }^{[}{ }^{4}$, Matthew O. Fillingim ${ }^{(16}$, Francois Forget ${ }^{7}$, \\ Heidi Fuqua Haviland $\mathbb{1}^{8}$, Matthew Golombek ${ }^{9}$, Steve Joy ${ }^{104}$, Philippe Lognonné ${ }^{10}$, Xinping Liu ${ }^{4}$, \\ Chloé Michaut ${ }^{11}$, Lu Pan ${ }^{10}{ }^{12}$, Cathy Quantin-Nataf ${ }^{12}$, Aymeric Spiga ${ }^{7,13}$, Sabine Stanley ${ }^{14,15}$, \\ Shea N. Thorne ${ }^{11}{ }^{1}$, Mark A. Wieczorek ${ }^{10}{ }^{16}$, Yanan Yu ${ }^{4}$, Suzanne E. Smrekar and William B. Banerdt $\mathbb{1}^{9}$
}

Magnetic fields provide a window into a planet's interior structure and evolution, including its atmospheric and space environments. Satellites at Mars have measured crustal magnetic fields indicating an ancient dynamo. These crustal fields interact with the solar wind to generate transient fields and electric currents in Mars's upper atmosphere. Surface magnetic field data play a key role in understanding these effects and the dynamo. Here we report measurements of magnetic field strength and direction at the InSight (Interior Exploration using Seismic Investigations, Geodesy and Heat Transport) landing site on Mars. We find that the field is ten times stronger than predicted by satellite-based models. We infer magnetized rocks beneath the surface, within $-150 \mathrm{~km}$ of the landing site, consistent with a past dynamo with Earth-like strength. Geological mapping and InSight seismic data suggest that much or all of the magnetization sources are carried in basement rocks, which are at least 3.9 billion years old and are overlain by between $200 \mathrm{~m}$ and $\mathbf{- 1 0} \mathrm{km}$ of lava flows and modified ancient terrain. Daily variations in the magnetic field indicate contributions from ionospheric currents at $120 \mathrm{~km}$ to $180 \mathrm{~km}$ altitude. Higher-frequency variations are also observed; their origin is unknown, but they probably propagate from even higher altitudes to the surface. We propose that the time-varying fields can be used to investigate the electrical conductivity structure of the martian interior.

T he Interior Exploration Using Seismic Investigations, Geodesy and Heat Transport (InSight) mission landed on Mars on 26 November 2018 at $4.50^{\circ} \mathrm{N}, 135.62^{\circ} \mathrm{E}$ in Elysium Planitia ${ }^{1,2}$. The InSight fluxgate magnetometer (IFG) is part of the Auxiliary Payload Sensor Suite (APSS) monitoring environmental conditions at the lander, with the primary purpose of accounting for sources of wind, temperature, pressure and magnetic field noise in the seismic data $^{3}$. The IFG is the first magnetometer deployed on the martian surface. It thus affords unique opportunities for magnetic fieldbased studies of the planet's interior, the ionosphere and the extent to which conditions in the solar wind affect the surface environment (Extended Data Fig. 1).

Mars orbiter missions have provided evidence for crustal magnetization acquired in an ancient global field ${ }^{4,5}$. However, surface measurements can identify weak and/or small-scale magnetic fields that are undetectable at satellite altitude but are needed to better constrain crustal magnetization, magnetizing field strength and geometry, and dynamo timing. Satellites have also monitored time-varying magnetic fields $\mathrm{s}^{6-9}$ that result from the interplanetary magnetic field
(IMF) and electric currents generated in the uppermost atmosphere (ionosphere). The amplitude of these fields at the martian surface is difficult to predict ${ }^{10}$. Surface observations from fixed ground stations provide essential information on the nature of ionospheric electric currents because temporal variations are not mixed with spatial variations as is the case for a moving satellite. Furthermore, a ground observatory can elucidate the types of magnetic 'weather' at the surface, including transient variations driven by either changing solar wind conditions or atmospheric processes. Here we report results from the first seven months of IFG data that shed new light on Mars's crustal magnetization and reveal the nature of time variations in the magnetic field at the martian surface.

The crustal magnetic field at the InSight landing site The IFG instrument, data acquisition strategy and data processing pipeline, specifically, the estimation and subtraction of fields not of martian origin, are detailed in Methods. The resulting IFG data show time variations superposed on a steady background field (Fig. 1 and Extended Data Fig. 2). Here we use a local coordinate system

'Department of Earth, Ocean and Atmospheric Sciences, University of British Columbia, Vancouver, British Columbia, Canada. ${ }^{2}$ Planetary Science Institute, Tucson, AZ, USA. 'Laboratoire de Planétologie et Géodynamique, UMR-CNRS 6112, Université de Nantes, Université d'Angers, CNRS, Nantes, France. ${ }^{4}$ Earth, Planetary and Space Sciences, University of California, Los Angeles, CA, USA. ${ }^{5}$ Cornell Center for Astrophysics and Planetary Science, Ithaca, NY, USA. ${ }^{6}$ Space Sciences Laboratory, University of California, Berkeley, CA, USA. ${ }^{7}$ Laboratoire de Météorologie Dynamique / Institut Pierre-Simon Laplace (LMD/IPSL), Sorbonne Université, Centre National de la Recherche Scientifique (CNRS), École Polytechnique, École Normale Supérieure (ENS), Campus Pierre et Marie Curie BC99, Paris, France. ${ }^{8}$ Marshall Space Flight Center, Huntsville, AL, USA. '`et Propulsion Laboratory, California Institute of Technology, Pasadena, CA, USA. ${ }^{10}$ Université Paris Diderot-Sorbonne Paris Cité, Institut de Physique du Globe de Paris, Paris, France. "Université de Lyon, École Normale Supérieure de Lyon, UCBL, CNRS, Laboratoire de Géologie de Lyon -Terre, Planètes, Environnement, Lyon, France. ${ }^{12}$ Université de Lyon, Université Claude Bernard Lyon 1, ENS de Lyon, CNRS, UMR 5276 Laboratoire de Géologie de Lyon -Terre, Planètes, Environnement, Villeurbanne, France. ${ }^{13}$ Institut Universitaire de France (IUF), Paris, France. ${ }^{14}$ Department of Earth and Planetary Sciences, Johns Hopkins University, Baltimore, MD, USA.

${ }^{15}$ Applied Physics Lab, Johns Hopkins University, Laurel, MD, USA. ${ }^{16}$ Université Côte d'Azur, Observatoire de la Côte d'Azur, CNRS, Laboratoire Lagrange, Nice, France. e-mail: cjohnson@eoas.ubc.ca 

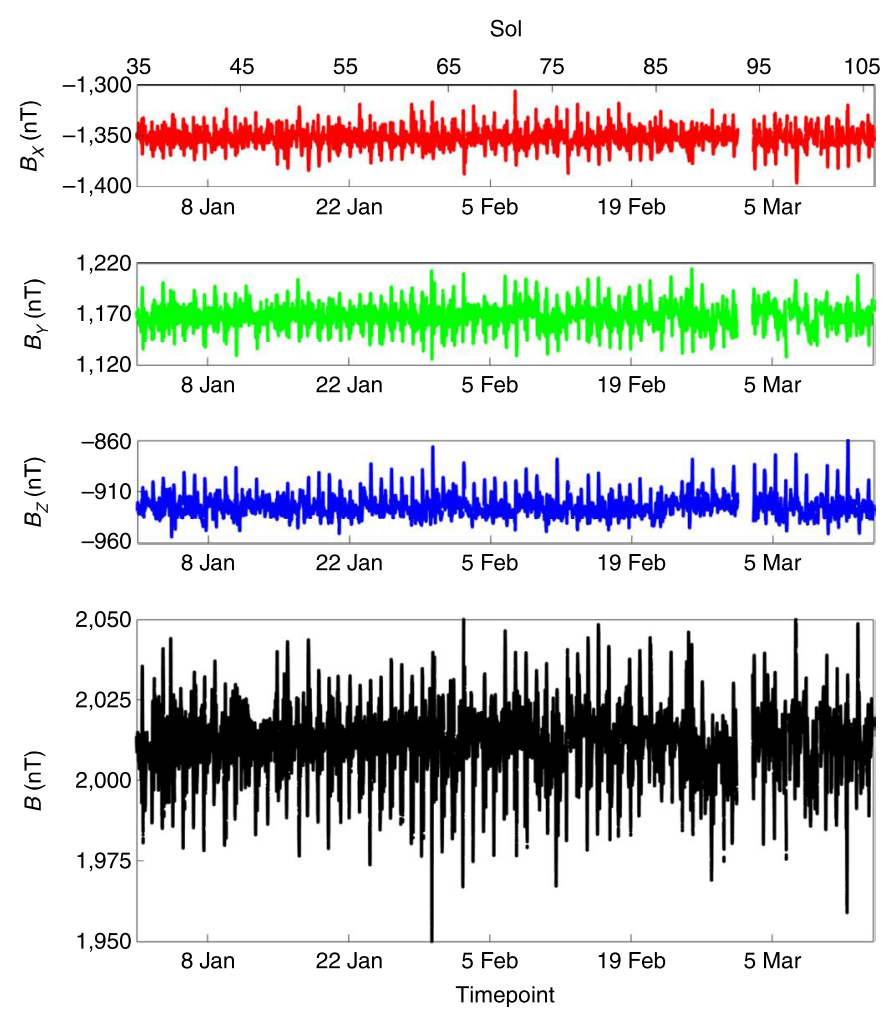

Fig. 1 | IFG data for sols 35-106. Magnetic field components ( $B_{X}$ (northward), $B_{Y}$ (eastward), $B_{Z}$ (downward)) and the total field $(B)$ in the local LL frame from 1 January 2019 to 15 March 2019 to show the average field and some of the observed time variations. Data gaps occurred due to safing of all APSS instruments, including IFG, at the time of Payload Auxiliary Electronics anomalies. An extended time series for sols 14-299 is shown in Extended Data Fig. 2.

(the InSight lander level (LL) frame) in which $X$ points north, $Y$ points east and $Z$ points down.

We take the observed average field as an estimate of the crustal field at the InSight landing site (Table 1). Uncertainties are described in Methods. The average crustal field at InSight is $\left(B_{X}, B_{Y}, B_{Z}\right)=(-1,353 \pm 19,1,168 \pm 83,-925 \pm 40)$ nT, yielding an average surface field strength of $2,013 \pm 53 \mathrm{nT}$ and direction southeast and upward (Table 1).

\section{Implications for magnetization}

Satellite observations allow estimates of the surface magnetic field from inversions. However, the maximum resolution in these models is on the order of the minimum spacecraft altitude. Data from the Mars Atmosphere and Volatile Evolution (MAVEN) mission ${ }^{5,11}$ have resulted in crustal field models that have higher spatial resolution than those previously available. Recent regional ${ }^{12}$ and global ${ }^{13}$ models that capture wavelengths greater than $\sim 150 \mathrm{~km}$ suggest surface fields strengths of 236-314 nT at the InSight landing site (Table 1 and Extended Data Fig. 3).

The IFG surface field is almost an order of magnitude larger and has a different dip than the satellite-derived estimates, implying substantial contributions from magnetization on length scales less than $\sim 150 \mathrm{~km}$. We investigate scenarios for the depths beneath the landing site at which that magnetization might be carried and the required magnetization.

The InSight landing site lies $\sim 500 \mathrm{~km}$ from the dichotomy boundary (Fig. 2a). Geological constraints on regional crustal structure indicate that beneath the landing site are Early Amazonian and
Table 1 | Martian surface magnetic field in the LL frame

$$
B_{X}(\mathrm{nT}) \quad B_{Y}(\mathrm{nT}) \quad B_{Z}(\mathrm{nT}) \quad B(\mathrm{nT}) \quad D\left(^{\circ}\right) \quad I\left(^{\circ}\right)
$$

\section{Spacecraft magnetic field measured pre-launch}

$\begin{array}{lllllll}\text { Average } & 552 & -430 & -22 & 700 & \mathrm{n} / \mathrm{a} & \mathrm{n} / \mathrm{a} \\ \text { Uncertainty } & 18 & 83 & 40 & 53 & \mathrm{n} / \mathrm{a} & \mathrm{n} / \mathrm{a} \\ \text { InSight surface field measurements } & & & & \\ \text { Average } & -1,353 & 1,168 & -925 & 2,013 & 139 & -27 \\ \text { s.d. } & 6 & 5 & 6 & \mathrm{n} / \mathrm{a} & \mathrm{n} / \mathrm{a} & \mathrm{n} / \mathrm{a} \\ \text { Combined error } & 19 & 83 & 40 & 53 & \mathrm{n} / \mathrm{a} & \mathrm{n} / \mathrm{a}\end{array}$

\section{Surface magnetic field predicted from recent satellite-based models}

$\begin{array}{lllllll}\text { Regional model }^{8} & -62 & 77 & -205 & 227 & 129 & -64\end{array}$

$\begin{array}{lllllll}\text { Global model }^{9} & -64 & 63 & -296 & 309 & 136 & -73\end{array}$

The average spacecraft field and its error are described in Methods. The mean surface field and its standard deviation from sols 14-299 (Extended Data Fig. 2) using เт 20:00-04:00 to minimize external field contributions. The combined error includes the uncertainties in the spacecraft field and the surface measurements. Declination, $D=\tan ^{-1}\left(B_{y}, B_{x}\right)$, and inclination, $l=\tan ^{-1}\left(B_{z} /\left(B_{x}{ }^{2}+B_{y}{ }^{2}\right)^{1 / 2}\right)$, give the azimuth clockwise from north and the dip of the field; ' $n / a^{\prime}$ ' values not used.

Hesperian age flows (3.6-1.5 billion years old (Gyr old)). These are $\sim 200-300 \mathrm{~m}$ thick on the basis of mapping the thickness of lava flows that embay large craters and on the maximum size of rocky ejecta craters ${ }^{14,15}$ (Fig. 2a). The Hesperian-Noachian transition unit (HNt, Fig. 2a) may have a subsurface extension to the northeast ${ }^{15,16}$ and may comprise part or all of the depth range $\sim 1-5 \mathrm{~km}$ below the InSight landing site. This unit is a complex mix of reworked highland materials and possible sediments ${ }^{16}$. The underlying basement is probably a continuation of unit $\mathrm{mNh}$ (Fig. 2a), dated at $3.9 \mathrm{Gyr}$ old, but may have an extended age from Early to Late Noachian ${ }^{15,16}$. Basement depths are inferred from an excavated phyllosilicate deposit in Kalpin crater northeast of the lander with a calculated uplift depth of $4-5 \mathrm{~km}$ (ref. ${ }^{15}$ ) and from the weak materials indicated by the lack of rocky ejecta craters larger than $\sim 2 \mathrm{~km}$ (ref. ${ }^{14}$ ). In parallel, receiver function analysis from the Seismic Experiment for Interior Structure (SEIS) instrument suggests the presence of an $8-11 \mathrm{~km}$ thick layer (Fig. 2) of altered or damaged crustal material ${ }^{17}$.

We inverted for the minimum magnetization required to explain the single station measurement of the surface field strength, using the formulation described by ref. ${ }^{18}$. The magnetization is assumed to be carried in a horizontal layer, extending from a burial depth, $Z_{\mathrm{U}}$, to a maximum depth, $Z_{\mathrm{L}}$, beneath the surface (that is, thickness of $Z_{\mathrm{L}}-Z_{\mathrm{U}}$ ). The direction of magnetization within the layer varies such that the minimum magnetization magnitude is found. The magnetized layer depth extent cannot be determined from a single station measurement, but we discuss plausible bounds on $Z_{\mathrm{U}}$ from the geological and seismological constraints on subsurface crustal structure and inferences regarding the dynamo timing. The most accepted timing scenario is one in which the dynamo terminated by $\sim 4.1$ billion years ago $(\mathrm{Ga})$ (for example, refs. ${ }^{4,19}$ ). A later dynamo has also been proposed ${ }^{20}$, but globally, the absence of crustal fields over Amazonian units argues against an Amazonian dynamo. Thus, $Z_{\mathrm{U}}$ must be at least $200 \mathrm{~m}$ (magnetized layer lies beneath the flows, but may include the transition unit) and is up to $4-5 \mathrm{~km}$ (magnetized layer is confined to a more-deeply buried Noachian basement) or even $10 \mathrm{~km}$ (no magnetization carried in the seismologically detected layer of altered material). The minimum magnetizations required for $Z_{\mathrm{U}}=200 \mathrm{~m}-10 \mathrm{~km}$ are $0.4-1.4 \mathrm{Am}^{-1}$ for $40-\mathrm{km}$-thick magnetized layers and $1.4-24 \mathrm{Am}^{-1}$ if the magnetization is confined to less than $1 \mathrm{~km}$ in thickness. The $40-\mathrm{km}$-thick layer buried at $200 \mathrm{~m}$ is a similar model geometry to that often used in inversions of satellite data (for example, refs. ${ }^{12,13}$ ), and those predict magnetization magnitudes of close to $1 \mathrm{Am}^{-1}$ on an $\sim 150 \mathrm{~km}$ spatial scale. The minimum magnetization required to explain the InSight data 
a

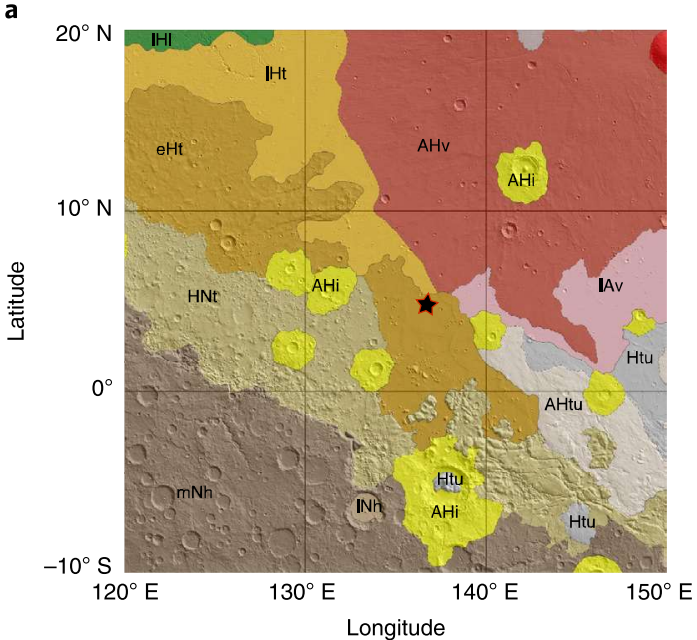

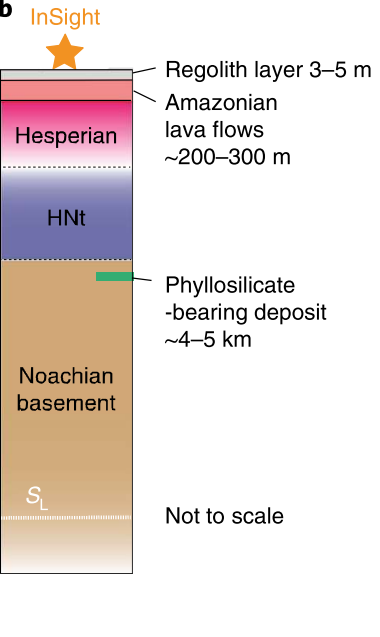

c

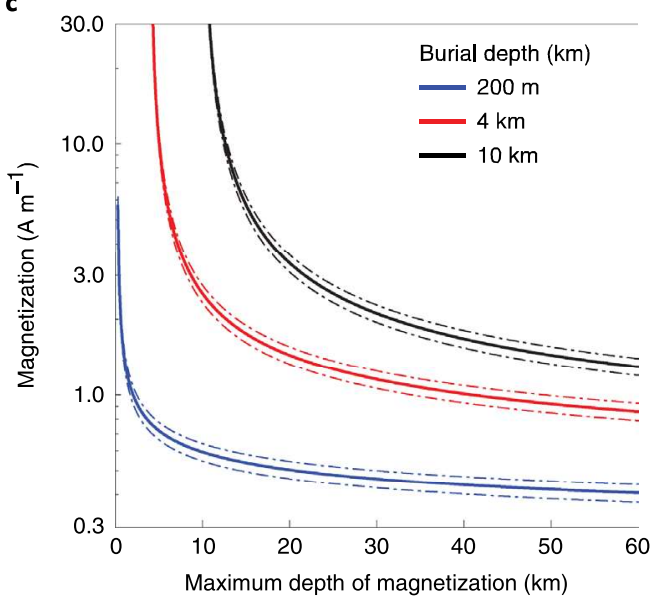

Fig. 2 | Regional geology and inferred magnetization. a, Geologic map ${ }^{39}$ after ref. ${ }^{16}$ with InSight landing site (star). The dichotomy boundary runs approximately northwest to southeast, following the boundary between the mNh and HNt units. Unit identifiers: mNh, middle Noachian highland; INh, late Noachian highland; HNt, Hesperian Noachian transition; eHt, early Hesperian transition; IHt, late Hesperian transition; AHv, Amazonian Hesperian volcanic; IAv, late Amazonian volcanic; Htu, Hesperian transition undivided; AHtu, Amazonian Hesperian transition undivided; AHi, Amazonian Hesperian impact. $\mathbf{b}$, The inferred stratigraphy beneath InSight. SEIS receiver function analysis based on InSight seismic data ${ }^{17}$ suggests the presence of altered or damaged crustal material up to $S_{L} \sim 10 \mathrm{~km}$. $S_{L}$, depth of the seismologically defined layer. c, Minimum magnetization required by the mean surface field strength of $2,013 \mathrm{nT}$ (solid) and the upper and lower $99 \%$ confidence intervals, that is, three times the combined error given in Table 1 (dashed). Free parameters are the burial depth (that is, the distance to the top of the magnetized layer) and maximum magnetization depth (that is, to the bottom). Three burial depths (different colours) are motivated by regional geological and seismic considerations.

is less than that required to explain the strong fields over the southern hemisphere ${ }^{18}$. Furthermore, the magnetizations are consistent with those estimated from the martian basaltic meteorites when magnetized in a $50 \mu \mathrm{T}$ field, that is, an Earth-like field strength ${ }^{21}$.

Current understanding suggests that the magnetization is predominantly carried in the Noachian basement. If that is in part the $3.9 \mathrm{Gyr}$ old $\mathrm{mNh}$ unit ${ }^{22}$, then the age of at least some of the magnetization postdates the canonical $4.1 \mathrm{Ga}$ dynamo termination ${ }^{4,19}$. Moreover, if the HNt unit lies beneath the lander and carries any magnetization, this would also be compatible with a dynamo operating after $4.1 \mathrm{Ga}$. Because these units are buried, no direct age estimate for the magnetization is available. However, the results offer the tantalizing suggestion of a longer-lived dynamo that would have implications for thermal evolution models for Mars and could be tested by future sample return missions such as Mars 2020 .

Additional constraints on magnetization depths could come from the power spectra of satellite magnetic field models ${ }^{23,24}$. In an $\sim 500 \mathrm{~km}$ region around the InSight landing site, the source depth was estimated to be $15 \mathrm{~km}$ (ref. ${ }^{24}$ ) on the basis of an early martian crustal field model. More recent approaches ${ }^{25}$ allow both the top and bottom of the source layer to be investigated, and investigations are under way (M.A.W., personal communication) to apply these to the recent martian global field model $^{13}$. In particular, $Z_{U}$ estimates will help address some of the open issues mentioned. Furthermore, although the magnetic sources probably reside in the crust, estimates for the local crustal thickness vary from 19 to $90 \mathrm{~km}$ $\left(\right.$ ref. $\left.{ }^{26}\right)$. SEIS data will play a key role in understanding where in the crust magnetization is carried by establishing an absolute crustal thickness value beneath the InSight landing site.

\section{Time-varying fields detected by IFG}

InSight is the first mission to make direct measurements of timevarying magnetic fields on the martian surface. Variations on different timescales and the processes that lead to those are given in Extended Data Fig. 4. Variations with daily, 26-day, and annual periods in the magnetic field have been observed in data collected by both Mars Global Surveyor (MGS) and MAVEN ${ }^{6-9}$ above the ionosphere, with a few observations at altitudes close to the ionospheric peak. Predicting how these signals will propagate through the ionosphere to the ground is difficult, and little literature exists on the expected amplitudes at the surface. Nonetheless, two recent studies have estimated that diurnal variations resulting from the combined effects of ionospheric currents and the draped IMF (Extended Data Fig. 4) will have typical amplitudes of a few to $\sim 20 \mathrm{nT}$ at the martian surface ${ }^{8,10}$. Signals with an $\sim 26$-day period reflecting synodic solar rotations and the associated changing polarity of the IMF every $\sim 13$ days (Extended Data Fig. 4) might be observed either directly or indirectly through their effects on ionospheric currents. Seasonal modulation of these daily and 26-day cycles may also occur related to changes in the ionospheric structure and heliocentric distance variations.

We find time-dependent signals dominated by daily variations (that is, within one sol, Fig. 3). The peak-to-peak amplitude of the daily variation on each component is typically less than $30 \mathrm{nT}$. Changes in these daily variations are expected because the ionospheric currents depend on neutral winds and the magnetic field at $\sim 120 \mathrm{~km}$ altitude. Neutral winds vary with season, and the magnetic field at ionospheric altitudes comprises the resultant of the steady crustal field ( 20-40 nT in each component at $120 \mathrm{~km}$ altitude) and the draped IMF, which varies with an $\sim 26$-day periodicity. Both neutral winds and the IMF can also vary on a sol-to-sol basis. We observe sol-to-sol variations (Fig. 3a,b) as well as longer timescale changes seen in the fields averaged over a few sols early (sols 50-59) and late (sols 218-226) in the mission so far. The occurrence of the peak fields in morning hours and the overall decrease in the peak field between sols $50-59$ and sols $218-226$ is consistent with the local time and Mars season dependencies predicted in ref. ${ }^{10}$. These measurements of external fields at a fixed ground location will be invaluable in understanding the geometry and time variations of ionospheric currents (for example, ref. ${ }^{10}$ ).

The PSD of time variations in the field clearly shows the daily variations and their harmonics (Fig. 3e). The current IFG time 
a
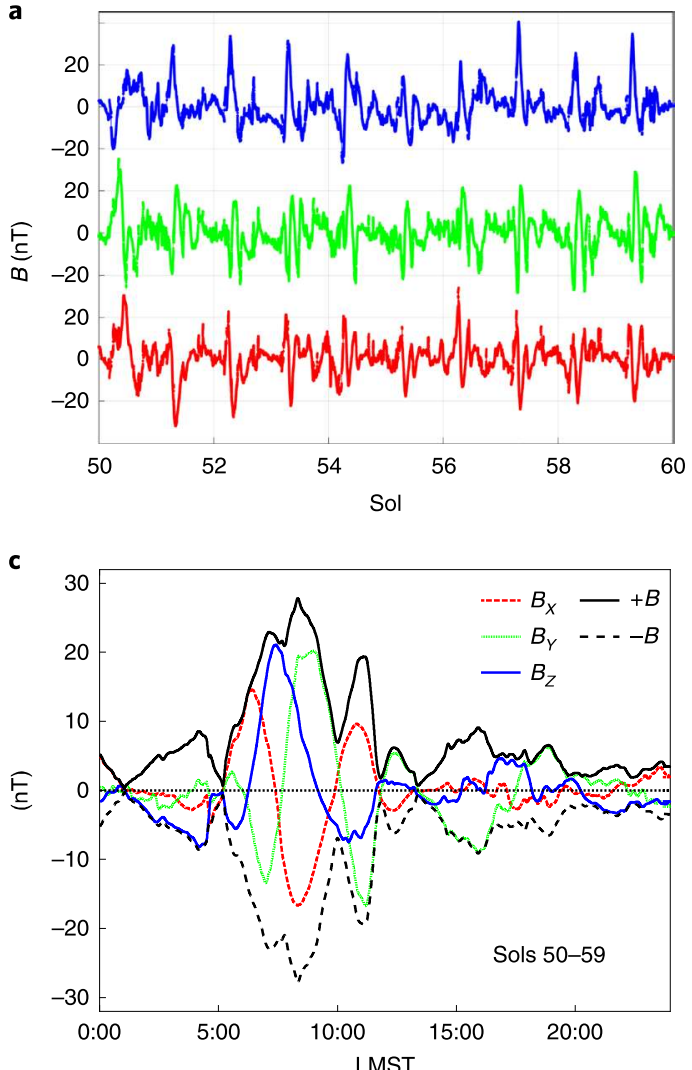

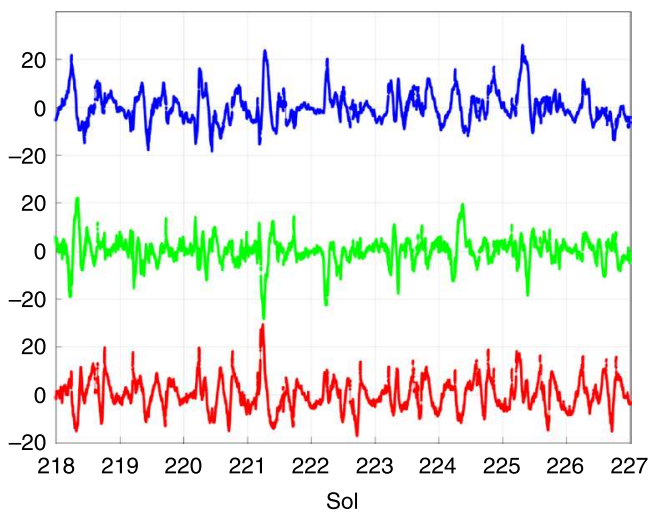

d

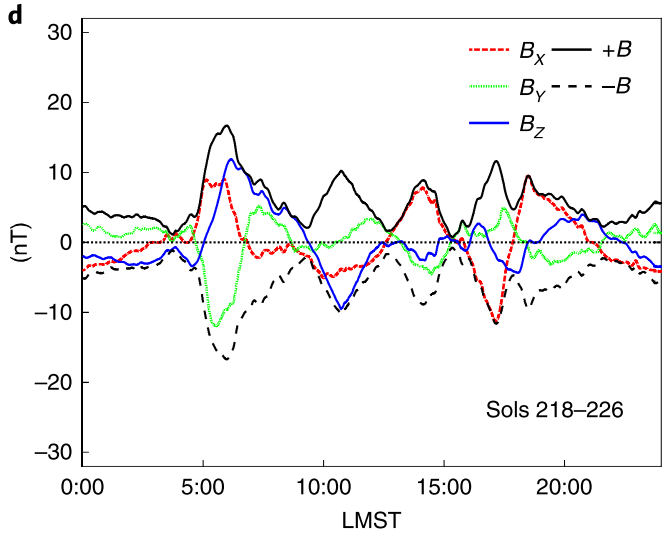

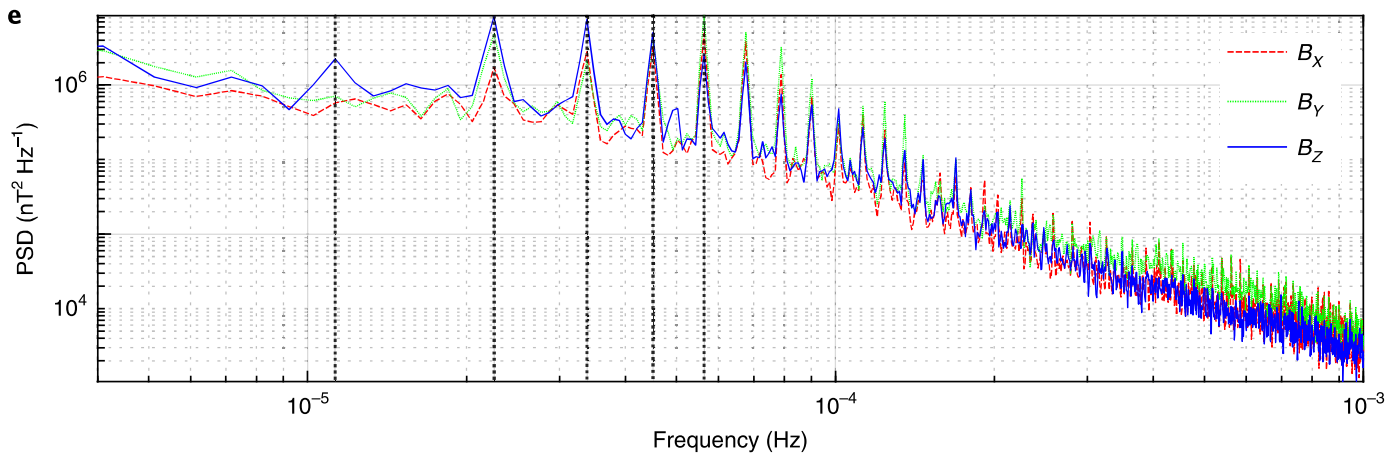

Fig. 3 | Time variations-daily signals. a,b, $B_{X}$ (red), $B_{Y}$ (green), $B_{Z}$ (blue) in the LL frame for sols $50-59$ (16 January $2019-26$ January 2019 ) (a) and sols 218-226 (8 July 2019-16 July 2019) (b). Each time series is demeaned and detrended over the ten-sol interval. c,d, The ten-sol-averaged daily signals corresponding to $\mathbf{a}$ and $\mathbf{b}$, respectively, versus local mean solar time (LMST), including amplitude ( $\pm B$ ). e, Power Spectral density (PSD) for the time interval sols 32-120 (29 December 2018-29 March 2019). Vertical dashed lines correspond to the daily period and its first four harmonics.

series is too short to see seasonal variations, and data gaps preclude any 26-day periodicity from being observed. At frequencies (f) above $\sim 0.5 \mathrm{mHz}$, the PSD falls off as $1 / f$, matching the preInSight predictions based on satellite data used in the noise model for SEIS operations ${ }^{27}$.

InSight has made the first detection of magnetic pulsations on the martian surface ${ }^{28}$. These are variations in the magnetic field with wave periods ranging from a second to a few minutes, and on Earth $^{29}$ and the Moon ${ }^{30}$, the occurrence of different types of pulsations implies specific physical processes above the atmosphere. InSight data show evidence for quasi-sinusoidal waves at around midnight with periods of $\sim 100 \mathrm{~s}$ (Fig. 4). Broadband pulsations with periods of a few minutes have also been observed in the late afternoon/early evening. We speculate that the former type of pulsations may be associated with oscillations in the electric current sheet in the induced magnetotail, and the latter might be induced either by the same mechanism or by oscillations on the flanks of the induced magnetosphere. Both the pulsations and the $1 / f$ dependence of the spectrum at frequencies substantially higher than 1 sol $^{-1}$ provide new information on the martian magnetic field environment and on ionospheric properties and processes. Before the InSight landing, it was unknown whether variations at these frequencies would be observed below the ionosphere. For example, aerobraking orbits on Venus Express and on MGS all showed weaker magnetic field variations below the ionosphere than within or above it, suggesting that the ionosphere might act as a conductive shield to such variations (for example, refs. ${ }^{3,31}$ )

Aperiodic signals are observed in the IFG data and are more challenging to investigate because of spacecraft activities (Extended Data Fig. 5 and Methods). As an example, we mention ongoing work to establish whether any daytime convective vortices potentially causing dust devils ${ }^{32}$ have associated magnetic 

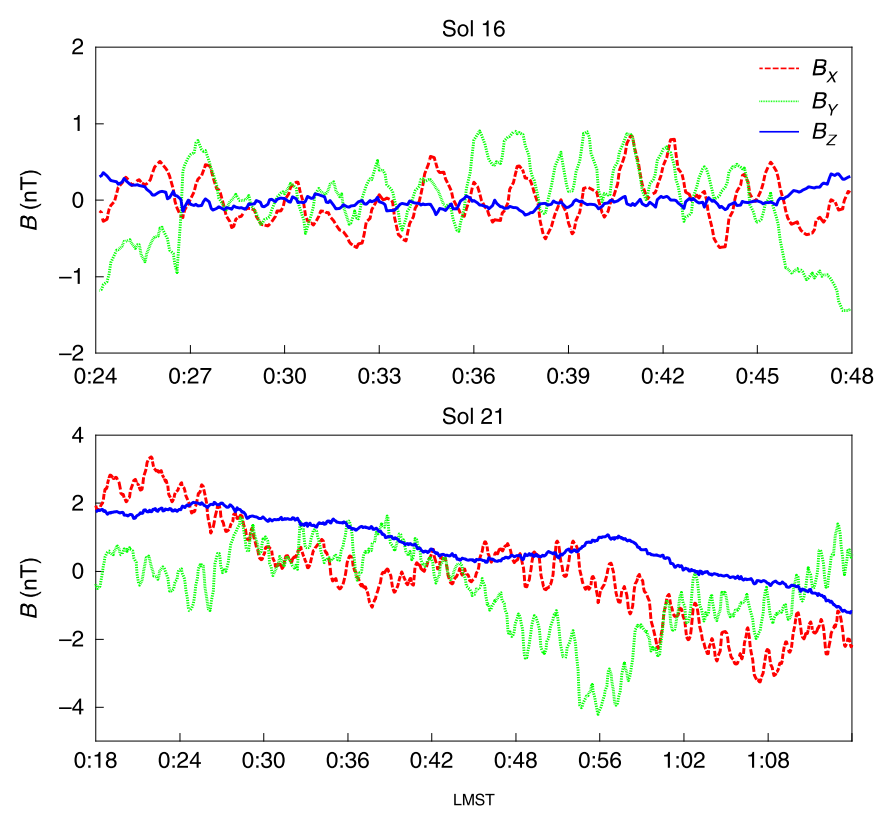

Fig. 4 | Pulsations. a,b, Two examples of detrended pulsations ( $B_{x}$ red; $B_{Y}$, green; $B_{z}$, blue), both occurring just after midnight LMST on sol $16 / 12$ December (a) and sol 21 / 17 December (b). Periodicities in both cases are $\sim 80 \mathrm{~s}$.

signals. In the first two months of the InSight mission, about 100 pressure drops deeper than $1.0 \mathrm{~Pa}$, indicative of such vortices close to InSight, have been identified, and calibrated IFG data at $20 \mathrm{~Hz}$ are available for 54 of these. At least 11 events (20\%) show a small change in the magnetic field, typically $<1 \mathrm{nT}$ in amplitude, correlated in time with the pressure drop (Extended Data Fig. 5). Triboelectric effects can lead to magnetic signatures if dust is in suspension ${ }^{33-35}$, thus, the observed signals might help discern dust-free vortices from dust devils. Although these early results are promising, more work is needed to confidently identify and interpret such signals.

\section{Future directions}

IFG data have provided the first direct evidence for localized magnetizations and time-varying fields on the surface of Mars, offering exciting new directions. The crustal magnetization results call for future low-altitude, near-surface or sample return studies, in particular to identify the age and carriers of magnetization. Diurnal variations will provide fundamental new constraints on ionospheric currents above the landing site and their dependence on the IMF and neutral winds. A longer time series of observations will elucidate whether there are seasonal variations in ionospheric currents that have observable effects at the surface. Investigations are under way to identify the origins of the magnetic pulsations reported here and to understand how they propagate to the surface. InSight and MAVEN together provide unique opportunities for concurrent surface and satellite observations. MAVEN has been measuring the plasma environment about Mars throughout the InSight mis$\operatorname{sion}^{5,11}$. To date, no clear correlation between IFG surface magnetic field perturbations and solar wind conditions has been observed. However, continued monitoring in this regard, in particular during the ascending phase of solar cycle 25 , will provide key constraints on when, how often and under what conditions solar wind conditions result in ground-based magnetic field signatures. For example, both interplanetary coronal mass ejection impacts and large solar flares can lead to increased ionospheric currents that may produce observable effects in the surface magnetic field. Flyovers of the InSight landing site by MAVEN in July and August 2019 occurred at altitudes as low as $\sim 150 \mathrm{~km}$ and will allow direct comparison of conditions at satellite altitude and time-varying fields measured on the ground.

Time-varying magnetic fields penetrate the subsurface to depths that depend on the interior electrical conductivity structure and the frequency content of the time variations (Fig. 5). The resulting induced fields, together with a priori knowledge of the geometry of the primary inducing field, or simultaneous direct measurements of this above the surface (for example, by MAVEN), yield information on electrical conductivity with depth. For Mars, mantle conductivities are likely in the range $0.1-1 \mathrm{~S} \mathrm{~m}^{-1}$ and higher in the lower mantle according to laboratory experiments conditions and a satellite study ${ }^{26,36-38}$ and can be probed by daily variations and their harmonics, as well as longer periods (Fig. 5). Investigations of the crust require higher frequencies and may be possible with the pulsation
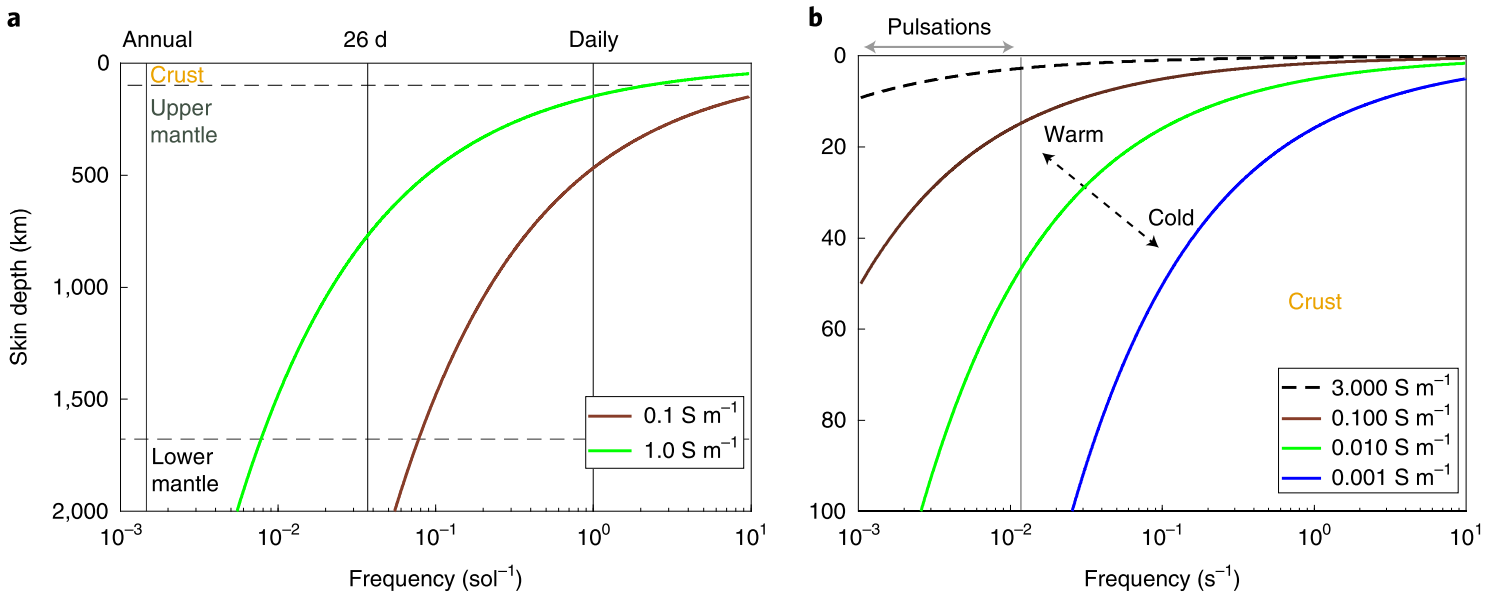

Fig. 5 | Toward interior electrical conductivity. $\mathbf{a}, \mathbf{b}$, Time-varying fields can probe the electrical conductivity structure of mantle (sol $\left.{ }^{-1}\right)(\mathbf{a})$, and crust $\left(\mathrm{s}^{-1}\right)$ (b). At the skin depth $(\delta)$, the amplitude of an electromagnetic wave $(f)$ is reduced by $1 / \mathrm{e}$, assuming a half-space conductivity $(\sigma), \delta \sim 500 /(\sigma)^{1 / 2}$. The Nyquist frequency of high-resolution IFG data is $10 \mathrm{~Hz}$. Expected upper-mantle conductivities are $0.1-1 \mathrm{~S} \mathrm{~m}^{-1}$ for different compositional and thermal models ${ }^{26}$. The electrical conductivity of the crust is probably lower ${ }^{26}$, although localized regions may have high conductivity due to thermal or compositional effects, in particular, the presence of water and/or hydrated minerals. 
signals. Thus, IFG data, including joint observations with MAVEN, afford unprecedented opportunities for probing interior thermal structure and volatile content that are complementary to investigations by the mission's science instruments and that address the major InSight objective of determining martian interior structure.

\section{Online content}

Any methods, additional references, Nature Research reporting summaries, source data, extended data, supplementary information, acknowledgements, peer review information; details of author contributions and competing interests; and statements of data and code availability are available at https://doi.org/10.1038/s41561020-0537-x.

Received: 29 August 2019; Accepted: 13 January 2020; Published online: 24 February 2020

\section{References}

1. Banerdt, W. B. et al. Initial results from the InSight mission on Mars. Nat. Geosci. https://doi.org/10.1038/s41561-020-0544-y (2020).

2. Golombek, M. et al. Geology of the InSight landing site on Mars Nat. Commun. https://doi.org/10.1038/s41467-020-14679-1 (2020).

3. Banfield, D. et al. InSight Auxiliary Payload Sensor Suite (APSS). Space Sci. Rev. 215, 4 (2018).

4. Acuna, M. H. et al. Global distribution of crustal magnetization discovered by the Mars global surveyor MAG/ER experiment. Science 284, 790-793 (1999).

5. Connerney, J. E. P. et al. First results of the MAVEN magnetic field investigation. Geophys. Res. Lett. 42, 8819-8827 (2015).

6. Luhmann, J. G., Russell, C. T., Brace, L. H. \& Vaisberg, O. L. in Mars (ed. George, M.) 1090-1134 (Univ. Arizona Press, 1992).

7. Brain, D., Bagenal, F., Acuña, M. H. \& Connerney, J. Martian magnetic morphology: contributions from the solar wind and crust. J. Geophys. Res. 108, 1424 (2003)

8. Mittelholz, A., Johnson, C. L. \& Lillis, R. J. Global-scale external magnetic fields at Mars measured at satellite altitude. J. Geophys. Res. Planets 112, 1243-1257 (2017).

9. Langlais, B., Civet, F. \& Thébault, E. In situ and remote characterization of the external field temporal variations at Mars. J. Geophys. Res. Planets 122, 110-123 (2017).

10. Lillis, R. J. et al. Modeling wind-driven ionospheric dynamo currents at Mars: expectations for InSight magnetic field measurements. Geophys. Res. Lett. 46, 5083-5091 (2019).

11. Jakosky, B. M. et al. The Mars atmosphere and volatile evolution (MAVEN) mission. Space Sci. Rev. 195, 3-48 (2015).

12. Mittelholz, A., Johnson, C. L. \& Morschhauser, A. A new magnetic field activity proxy for Mars from MAVEN data. Geophys. Res. Lett. 45, 5899-5907 (2018).

13. Langlais, B., Thébault, E., Houliez, A. \& Purucker, M. E. A new model of the crustal magnetic field of Mars using MGS and MAVEN. J. Geophys. Res. Planet 124, 1542-1569 (2019).

14. Golombek, M. et al. Geology and physical properties investigations by the InSight lander. Space Sci. Rev. 214, 84 (2018).

15. Pan, L. et al. Crust stratigraphy and heterogeneities of the first kilometers at the dichotomy boundary in western Elysium Planitia and implications for InSight lander. Icarus 338, 113511 (2020).
16. Tanaka, K. L. et al. Geologic Map of Mars Scientific Investigations Map 3292 (USGS, 2014)

17. Lognonné, P. et al. Constraints on the shallow elastic and anelastic structure of Mars from InSight seismic data. Nat. Geosci. https://doi.org/10.1038/ s41561-020-0536-y (2020).

18. Parker, R. L. Ideal bodies for Mars magnetics. J. Geophys. Res. 108 , 5006 (2003).

19. Lillis, R. J., Robbins, S., Manga, M., Halekas, J. S. \& Frey, H. V. Time history of the martian dynamo from crater magnetic field analysis. J. Geophys. Res. Planets 118, 1488-1511 (2013).

20. Schubert, G., Russell, C. T. \& Moore, W. B. Geophysics: timing of the martian dynamo. Nature 408, 666-667 (2000)

21. Gattacceca, J. et al. Martian meteorites and martian magnetic anomalies: a new perspective from NWA 7034. Geophys. Res. Lett. 41, 4859-4864 (2014).

22. Head, J. W., Kreslavsky, M. A. \& Pratt, S. Northern lowlands of Mars: evidence for widespread volcanic flooding and tectonic deformation in the Hesperian Period. J. Geophys. Res. 107, 5003 (2002).

23. Voorhies, C. V., Sabaka, T. J. \& Purucker, M. On magnetic spectra of Earth and Mars. J. Geophys. Res. 107, 5034 (2002).

24. Lewis, K. W. \& Simons, F. J. Local spectral variability and the origin of the martian crustal magnetic field. Geophys. Res. Lett. 39, L18201 (2012).

25. Wieczorek, M. A. Strength, depth, and geometry of magnetic sources in the crust of the Moon from localized power spectrum analysis. J. Geophys. Res. Planets 123, 291-316 (2018).

26. Smrekar, S. E. et al. Pre-mission InSights on the Interior of Mars. Space Sci. Rev. 215, 3 (2018)

27. Mimoun, D. et al. The noise model of the SEIS seismometer of the InSight mission to Mars. Space Sci. Rev. 211, 383-428 (2017).

28. Chi, P. J. et al. Magnetic pulsations on martian surface: initial results from InSight fluxgate magnetometer. In Proc. 50th Lunar Planet. Sci. Conf. Abstract 1752 (Lunar and Planetary Institute, Houston, 2019).

29. Saito, T. Geomagnetic pulsations. Space Sci. Rev. 10, 319-412 (1969).

30. Chi, P. J., Russell, C. T., Wei, H. Y. \& Farrell, W. M. Observations of narrowband ion cyclotron waves on the surface of the Moon in the terrestrial magnetotail. Planet. Space Sci. 89, 21-28 (2013).

31. Zhang, T. L., Baumjohann, W., Russell, C. T., Luhmann, J. G. \& Xiao, S. D. Weak, quiet magnetic fields seen in the Venus atmosphere. Sci. Rep. 6 , 23537 (2016).

32. Banfield, D. et al. The atmosphere of Mars as observed by InSight. Nat. Geosci. https://doi.org/10.1038/s41561-020-0534-0 (2020).

33. Jackson, T. L. \& Farrell, W. M. Electrostatic fields in dust devils: an analog to Mars. IEEE Trans. Geosci. Remote Sens. 44, 2942-2949 (2006).

34. Farrell, W. M. Electric and magnetic signatures of dust devils from the 2000-2001 MATADOR desert tests. J. Geophys. Res. 109, E03004 (2004)

35. Kurgansky, M. V., Baez, L. \& Ovalle, E. M. A simple model of the magnetic emission from a dust devil. J. Geophys. Res. 112, E11008 (2007)

36. Vacher, P. \& Verhoeven, O. Modelling the electrical conductivity of iron-rich minerals for planetary applications. Planet. Space Sci. 55, 455-466 (2007).

37. Civet, F. \& Tarits, P. Electrical conductivity of the mantle of Mars from MGS magnetic observations. Earth Planet. Space 66, 85 (2014).

38. Verhoeven, O. \& Vacher, P. Laboratory-based electrical conductivity at martian mantle conditions. Planet. Space Sci. 134, 29-35 (2016).

39. Christensen, P. R. et al. JMARS-A Planetary GIS. IN22A-06 (AGU Fall Meeting, 2009) 